\title{
INEQUALITIES FOR GENERALIZED PARAMETRIC MARCINKIEWICZ INTEGRALS ALONG POLYNOMIAL COMPOUND CURVES
}

\author{
FENG LiU, Zunwei Fu AND LiguANG WANG
}

\begin{abstract}
Under some weaker size conditions assumed on the integral kernels both on the unit sphere and in the radial directions, the sharp $L^{p}$ boundedness was proved for the generalized parametric Marcinkiewicz integrals along polynomial compound curves via an extrapolation argument. As applications, the corresponding results for generalized parametric Marcinkiewicz integral operators related to the Littlewood-Paley $g_{\lambda}^{*}$-functions and area integrals are also established.
\end{abstract}

Mathematics subject classification (2010): 42B20, 42B25, 42B99.

Keywords and phrases: Generalized parametric Marcinkiewicz integral, rough kernel, polynomial compound curve, Triebel-Lizorkin space, extrapolation.

\section{REFERENCES}

[1] H. Al-QAssem, L. C. Cheng And Y. Pan, On rough generalized parametric Marcinkiewicz integrals, J. Math. Inequal., 11, 3 (2017), 763-780.

[2] H. Al-QAssem, L. C. Cheng And Y. Pan, Boundedness of rough integral operators on TriebelLizorkin spaces, Publ. Mat., 56, 1 (2012), 261-277.

[3] A. Al-Salman, H. Al-Qassem, L. C. Cheng And Y. Pan, $L^{p}$ bounds for the function of Marcinkiewicz, Math. Res. Lett., 9, 5 (2002), 697-700.

[4] H. AL-QASSEM AND Y. PAN, On certain estimates for Marcinkiewicz integrals and extrapolation, Collect. Math., 60, 2 (2009), 123-145.

[5] J. CHEN, D. FAN AND Y. YING, Singular integral operators on function spaces, J. Math. Anal. Appl., 276, 1 (2002), 691-708.

[6] Y. Ding, D. FAN AND Y. PAN, $L^{p}$-boundedness of Marcinkiewicz integrals with Hardy space function kernel, Acta Math. Sin. (Engl. Ser.), 16, 4 (2000), 593-600.

[7] Y. Ding, D. FAn AND Y. PAN, On the $L^{p}$ boundedness of Marcinkiewicz integrals, Michigan Math. J., 50, 1 (2002), 17-26.

[8] Y. Ding, Q. Xue And K. Yabuta, Boundedness of the Marcinkiewicz integrals with rough kernel associated to surfaces, Tohoku Math. J., 62, 2(2010), 233-262.

[9] D. FAN AND H. Wu, On the generalized Marcinkiewicz integral operators with rough kernels, Canad. Math. Bull., 54, 1(2011), 100-112.

[10] L. GRAFAKOS AND A. STEFANOV, $L^{p}$ bounds for singular integrals and maximal singular integral with rough kernels, Indiana Univ. Math. J., 47, 1(1998), 455-469.

[11] L. HöRmANDER, Estimates for translation invariant operators in $L^{p}$ spaces, Acta Math., 104, 10(1960), 93-104.

[12] Y. JiAng AND S. LU, $L^{p}$ boundedness of a class of maximal singular integral operators, Acta Math. Sin. (Chin. Ser.), 35, 1 (1992), 63-72.

[13] H. V. LE, Singular integrals with mixed homogeneity in Triebel-Lizorkin spaces, J. Math. Anal. Appl., 345, 1(2008), 903-916.

[14] F. LiU, On singular integrals associated to surfaces, Tohoku Math. J., 66, 1(2014), 1-14.

[15] F. LIU, Integral operators of Marcinkiewicz type on Triebel-Lizorkin spaces, Math. Nachr., 290, 1(2017), 75-96. 
[16] F. LiU, A note on Marcinkiewicz integrals associated to surfaces of revolution, J. Austral. Math. Soc., 104, 1(2018), 380-402.

[17] F. LiU, A note of Littlewood-Paley functions on Triebel-Lizorkin spaces, Bull. Korean Math. Soc., 55, 2(2018), 659-672.

[18] F. LiU, A note on generalized parametric Marcinkiewicz integrals, Bull. Korean Math. Soc., 56, 5(2019), 1099-1115.

[19] F. LiU, Z. FU AND S. JHANG, Boundedness and continuity of Marcinkiewicz integrals associated to homogeneous mappings on Triebel-Lizorkin spaces, Front. Math. China, 14, 1(2019), 95-122.

[20] F. LiU, Z. Fu, Y. Zheng AND Q. YUAN, A rough Marcinkiewicz integral along smooth curves, J. Nonlinear Sci. Appl., 9, 1(2016), 4450-4464.

[21] F. LiU AND H. WU, On Marcinkiewicz integrals associated to compound mappings with rough kernels, Acta Math. Sin. (Engl. Ser.), 30, 7(2014), 1210-1230.

[22] F. LIU AND H. WU, $L^{p}$ bounds for Marcinkiewicz integrals associated to homogeneous mappings, Monatsh. Math., 181, 4(2016), 875-906.

[23] F. Liu AND D. Zhang, Parabolic Marcinkiewicz integrals associated to polynomials compound curves and extrapolation, Bull. Korean Math. Soc., 52, 3(2015), 771-788.

[24] M. Sakamoto And K. Yabuta, Boundedness of Marcinkiewicz, functions, Studia Math., 135, 1(1999), 103-142.

[25] S. SAto, Estimates for singular integrals along surfaces of revolution, J. Austral. Math. Soc., 86, 2(2009), 413-430.

[26] E. M. Stein, On the function of Littlewood-Paley, Lusin and Marcinkiewicz, Trans. Amer. Math. Soc., 88, 2(1958), 430-466.

[27] K. YABUTA, Triebel-Lizorkin space bounddedness of Marcinkiewicz integrals associated to surfaces, Appl. Math. J. Chinese Univ., 30, 4(2015), 418-446. 